\title{
A Filled Function Method Dominated by Filter for Nonlinearly Global Optimization
}

\author{
Wei Wang, ${ }^{1}$ Xiaoshan Zhang, ${ }^{2}$ and ${\mathrm{Min} \mathrm{Li}^{1}}^{1}$ \\ ${ }^{1}$ Department of Mathematics, East China University of Science and Technology, Shanghai 200237, China \\ ${ }^{2}$ Shangnan Middle School, South Campus, Shanghai 200123, China \\ Correspondence should be addressed to Wei Wang; wangwei@ecust.edu.cn
}

Received 18 August 2014; Revised 30 December 2014; Accepted 8 January 2015

Academic Editor: George Fikioris

Copyright (c) 2015 Wei Wang et al. This is an open access article distributed under the Creative Commons Attribution License, which permits unrestricted use, distribution, and reproduction in any medium, provided the original work is properly cited.

\begin{abstract}
This work presents a filled function method based on the filter technique for global optimization. Filled function method is one of the effective methods for nonlinear global optimization, since it can effectively find a better minimizer. Filter technique is applied to local optimization methods for its excellent numerical results. In order to optimize the filled function method, the filter method is employed for global optimizations in this method. A new filled function is proposed first, and then the algorithm and its properties are proved. The numerical results are listed at the end.
\end{abstract}

\section{Introduction}

In this paper, we consider the following nonlinearly global optimization:

$$
\min \left\{f(x): x \in R^{n}\right\}
$$

where $f: R^{n} \rightarrow R$. Global optimization problem has been developed rapidly in recent years. One of the different significant characteristics of the global optimization from local optimization is that it has more than one minimum point. It obviously makes the problem difficult to resolve. In the search of global optimization, we will face two difficulties: the first is how to find a better minimizer from a known local one; the second is how to judge whether the current local minimizer is a global optimum or not. The filled function is one of the valuable methods for the first difficulty. The filled function was first proposed by Ge and Qin [1,2]. A great deal of efforts has been made by successive scholars and experts [3-6], which makes filled function algorithm develop rapidly. The filled function method has been applied in many practical fields [7].

The filter method was firstly proposed by Fletcher and Leyffer $[8,9]$ for solving nonlinearly local optimization. Because of its excellent numerical results, many researchers show their interest in it $[10,11]$. In order to optimize the filled function method, the filter method is employed for a global optimization in this paper. So we will propose a filter-filled function method for problem (1).

This paper is organized as follows. In Section 2, we first give some assumptions and marks and then some definitions of filled function and the filter method are introduced. In Section 3, a new filled function is proposed. The algorithm for problem (1) and its properties are discussed in Section 4. In the last section, we will list the numerical tests.

\section{Preliminaries}

Our purpose is to find a global minimizer of problem (1). We make the following assumptions for the objective function $f(x)$ throughout the paper.

(A1) $f(x)$ is continuously differentiable in $R^{n}$.

(A2) $f(x) \rightarrow \infty$, as $\|x\| \rightarrow \infty$; namely, $f(x)$ is a coercive function.

(A3) $f(x)$ has only a finite number of minimal function values.

According to (A2), we just need to consider the problem

$$
\min \{f(x): x \in X\},
$$

where $X \subset R^{n}$ is a closed and bounded domain and contains all of the local and global minimizers of the objective $f(x)$. 
For simplicity, we introduce some marks.

$L(P)$ and $G(P)$, respectively, stand for the set of local and global minimizers.

Let $x^{*}$ be a current local minimizer of $f(x)$. It can be obtained by a classical algorithm, such as Newton's method or steepest descent method.

The radius of $X$ is defined as

$$
D=\max _{x, y \in X}\|x-y\| \text {. }
$$

Consider $S_{1}\left(x^{*}\right)=\left\{x \mid f(x) \geq f\left(x^{*}\right), x \in X \backslash\left\{x^{*}\right\}\right\}$; we call $S_{1}\left(x^{*}\right)$ the high level set at $x^{*}$.

Consider $S_{2}\left(x^{*}\right)=\left\{x \mid f(x)<f\left(x^{*}\right), x \in X\right\}$; we call $S_{2}\left(x^{*}\right)$ the low level set at $x^{*}$.

We let int $X$ denote the interior set of $X$ and let $\partial X$ denote the boundary set of $X$.

For any $x^{*} \in$ int $X$, its neighborhood is denoted by $U\left(x^{*}, \delta\right)=\left\{x \mid\left\|x-x^{*}\right\|<\delta\right\}$, and its deleted neighborhood by $U^{0}\left(x^{*}, \delta\right)=\left\{x \mid\left\|x-x^{*}\right\|<\delta, x \neq x^{*}\right\}$.

The following is the definition of the filled function for solving problem (1).

Definition 1. Assume that $x^{*}$ is a local minimizer of the original objective function $f(x)$; a function $p\left(x, x^{*}\right)$ is called filled function of $f(x)$ at $x^{*}$, if

(i) $x_{1}^{*}$ is a strict local maximizer of $p\left(x, x^{*}\right)$,

(ii) $p\left(x, x^{*}\right)$ has no stationary point in the high lever set $S_{1}\left(x^{*}\right)$,

(iii) $f\left(x^{*}\right)$ is not a global minimum, that is, $x^{*} \notin G(P)$, then there exists a point $x_{0}^{*} \in S_{2}\left(x^{*}\right)$, such that $x_{0}^{*}$ is a minimizer of $p\left(x, x^{*}\right)$.

Filter technique is usually applied to local optimization methods. In order to optimize the filled function method, we employ it for global optimization in this paper because of its excellent numerical results. The filter mainly consists of two competitive objective functions $\phi(x)$ and $\psi(x)$, which are denoted by $(\phi(x), \psi(x))$. Now we borrow the concept of domination from multiobjective optimization to give a list of concept of filters.

Definition 2. A point $x_{k}$ is said to dominate another point $x_{l}$ if and only if both $\phi\left(x_{k}\right) \leq \phi\left(x_{l}\right)$ and $\psi\left(x_{k}\right) \leq \psi\left(x_{l}\right)$ hold.

Definition 3. A filter is a list of pairs $\left(\phi\left(x_{k}\right), \psi\left(x_{k}\right)\right)$ such that no point dominates any other. Namely, for the two inequalities $\phi\left(x_{k}\right) \leq \phi\left(x_{l}\right)$ and $\psi\left(x_{k}\right) \leq \psi\left(x_{l}\right)$, only one of them is true.

We use $F_{k}$ to denote the set of $x_{l}(l \leq k)$ such that $\left(\phi\left(x_{l}\right), \psi\left(x_{l}\right)\right)$ is an entry in the current filter. A point $x_{k+1}$ is said to be "acceptable for the filter" if and only if

$$
\begin{array}{r}
\phi\left(x_{k+1}\right) \leq(1-\gamma) \phi\left(x_{l}\right) \text { or } \\
\psi\left(x_{k+1}\right) \leq(1-\eta) \psi\left(x_{l}\right), \\
\exists x_{l} \in F_{k}
\end{array}
$$

holds, where $\gamma, \eta \in(0,1)$ and are closed to zero.
We may also "update the filter," which means that the pair $\left(\phi\left(x_{k+1}\right), \psi\left(x_{k+1}\right)\right)$ is added to the list of pairs in the filter, and at the same time any pair dominated by $\left(\phi\left(x_{k+1}\right), \psi\left(x_{k+1}\right)\right)$ in the filter is removed. Namely, we have

$$
\begin{gathered}
F_{k+1}=F_{k} \cup\left\{x_{k+1}\right\} \backslash\left\{x_{l} \in F_{k} \mid \phi\left(x_{k+1}\right) \leq \phi\left(x_{l}\right),\right. \\
\left.\psi\left(x_{k+1}\right) \leq \psi\left(x_{l}\right)\right\} .
\end{gathered}
$$

By the concepts above, we can define a filter as a criterion for accepting or rejecting a trial step. In this paper, the original objective function $f(x)$ will replace $\phi(x)$, and the filled function $p\left(x, x^{*}\right)$ will replace $\psi(x)$. Additionally, $|F|$ stands for the number of elements in the set $F$.

\section{A New Filled Function}

In this paper, we construct a new filled function with one parameter for problem (1). We suppose that a local minimizer $x^{*}$ of problem (1) has been obtained; $\rho>0$ is a parameter. The filled function is defined as

$$
p\left(x, x^{*}, \rho\right)=-\left\|x-x^{*}\right\|^{2}+\frac{\rho}{1+\min ^{2}\left\{0, f(x)-f\left(x^{*}\right)\right\}} .
$$

According to assumption (A1), it can be easily proved that the following conclusion is true.

Theorem 4. The filled function $p\left(x, x^{*}, \rho\right)$ is continuously differentiable in $R^{n}$.

Now we investigate the filled properties of the function $p\left(x, x^{*}, \rho\right)$.

Theorem 5. If $x^{*}$ is a local minimum of problem (1), then $x^{*}$ is a strict maximizer of $p\left(x, x^{*}, \rho\right)$ for any $\rho>0$.

Proof. Consider $\forall x \in X$ and $x \neq x^{*}$,

$$
\begin{aligned}
p\left(x, x^{*}, \rho\right) & =-\left\|x-x^{*}\right\|^{2}+\frac{\rho}{1+\min ^{2}\left\{0, f(x)-f\left(x^{*}\right)\right\}} \\
& <\frac{\rho}{1+\min ^{2}\left\{0, f(x)-f\left(x^{*}\right)\right\}} \\
& \leq \rho=p\left(x^{*}, x^{*}, \rho\right) .
\end{aligned}
$$

Theorem 6. The function $p\left(x, x^{*}, \rho\right)$ has no stationary point in the region $S_{1}\left(x^{*}\right)$.

Proof. Let $x \in S_{1}\left(x^{*}\right)$; that is, $f(x) \geq f\left(x^{*}\right)$ and $x \neq x^{*}$; then

$$
p\left(x, x^{*}, \rho\right)=-\left\|x-x^{*}\right\|^{2}+\rho .
$$

Obviously, it has no stationary point except $x^{*}$.

Theorem 7. Suppose $x^{*} \in L(P)$, but $x^{*} \notin G(P)$; then for large enough $\rho>0, p\left(x, x^{*}, \rho\right)$ has a local minimizer in the region $S_{2}\left(x^{*}\right)$. 
Proof. Consider $\forall x \in S_{2}\left(x^{*}\right), p\left(x, x^{*}, \rho\right)=-\left\|x-x^{*}\right\|^{2}+\rho /(1+$ $\left.\left[f(x)-f\left(x^{*}\right)\right]^{2}\right)$.

Since $x^{*} \notin G(P)$, there must exist an $x^{G} \in G(P)$ such that $f\left(x^{G}\right)<f\left(x^{*}\right)$. According to assumption (A3), there is an $\epsilon>0$ which makes $f\left(x^{G}\right)+\epsilon<f\left(x^{*}\right)$.

Let $L_{\epsilon}=\left\{x \mid f(x)=f\left(x^{G}\right)+\epsilon\right\}$. It is easy to see

$$
f\left(x^{G}\right)-f\left(x^{*}\right)<f(x)-f\left(x^{*}\right)<0, \quad \forall x \in L_{\epsilon} .
$$

In the following, we will show that, $\forall x \in L_{\epsilon}, p\left(x^{G}, x^{*}, \rho\right)<$ $p\left(x, x^{*}, \rho\right)$, while $\rho$ is large enough.

Consider two cases for $x \in L_{\epsilon}$ : (i) $\left\|x^{G}-x^{*}\right\| \geq\left\|x-x^{*}\right\|$; (ii) $\left\|x^{G}-x^{*}\right\|<\left\|x-x^{*}\right\|$.

(i) We have $-\left\|x^{G}-x^{*}\right\| \leq-\left\|x-x^{*}\right\|$ and $\left[f\left(x^{G}\right)-f\left(x^{*}\right)\right]^{2}>$ $\left[f(x)-f\left(x^{*}\right)\right]^{2}$ by expression (9). Therefore,

$$
\begin{aligned}
-\left\|x^{G}-x^{*}\right\|^{2} & +\frac{\rho}{1+\left[f\left(x^{G}\right)-f\left(x^{*}\right)\right]^{2}} \\
& <-\left\|x-x^{*}\right\|^{2}+\frac{\rho}{1+\left[f(x)-f\left(x^{*}\right)\right]^{2}} .
\end{aligned}
$$

That is, $p\left(x^{G}, x^{*}, \rho\right)<p\left(x, x^{*}, \rho\right), x \in L_{\epsilon}$.

(ii) Since

$$
\begin{gathered}
\left\|x^{G}-x^{*}\right\|-\left\|x-x^{*}\right\|<0, \\
\frac{1}{1+\left[f\left(x^{G}\right)-f\left(x^{*}\right)\right]^{2}}-\frac{1}{1+\left[f(x)-f\left(x^{*}\right)\right]^{2}}<0,
\end{gathered}
$$

the expression

$$
\begin{gathered}
\rho\left[\frac{1}{1+\left[f\left(x^{G}\right)-f\left(x^{*}\right)\right]^{2}}-\frac{1}{1+\left[f(x)-f\left(x^{*}\right)\right]^{2}}\right] \\
\quad<\left\|x^{G}-x^{*}\right\|-\left\|x-x^{*}\right\|
\end{gathered}
$$

surely holds for $\rho>0$ larger enough.

The inequality (12) is equivalent to (10). So $p\left(x^{G}, x^{*}, \rho\right)<$ $p\left(x, x^{*}, \rho\right)\left(x \in L_{\epsilon}\right)$ holds.

Now we set $\mathscr{L}_{\epsilon}=\left\{x \mid f(x) \leq f\left(x^{G}\right)+\epsilon\right\}$ and it is a compact set for $\overline{f(x)}$ is continuous. The function $p\left(x, x^{*}, \rho\right)$ is continuous on $\mathscr{L}_{\epsilon}$; thus it has a minimizer $x_{0}^{*} \in \mathscr{L}_{\epsilon}$. That means

$$
\min _{x \in \underline{\mathscr{L}_{\epsilon}}} p\left(x, x^{*}, \rho\right)=p\left(x_{0}^{*}, x^{*}, \rho\right) .
$$

$$
\begin{aligned}
& \text { Because } x^{G} \in \underline{\mathscr{L}_{\epsilon}}, L_{\epsilon} \subseteq \underline{\mathscr{L}_{\epsilon}} \text {, and } \\
& p\left(x_{0}^{*}, x^{*}, \rho\right) \leq p\left(x^{G}, x^{*}, \rho\right)<p\left(x, x^{*}, \rho\right), \quad \forall x \in L_{\epsilon},
\end{aligned}
$$

we can learn $x_{0}^{*} \in \mathscr{L}_{\epsilon} \backslash L_{\epsilon}$. But the set $\mathscr{L}_{\epsilon} \backslash L_{\epsilon} \subseteq S_{2}\left(x^{*}\right)$ on the basis of $f\left(x^{G}\right)+\epsilon<f\left(x^{*}\right)$; we have got $x_{0}^{*} \in S_{2}\left(x^{*}\right)$.

\section{Filter-Filled Function Algorithm and Its Properties}

In this section the search directions and their properties will be discussed first. Then the algorithm will be presented.

With the definition of $p\left(x, x^{*}, \rho\right), \nabla p\left(x, x^{*}, \rho\right)=-2(x-$ $\left.x^{*}\right)$ at $x \in S_{1}\left(x^{*}\right)$, so the following theorem holds.

When $\nabla f(x) \neq 0$ or $\nabla p\left(x, x^{*}, \rho\right) \neq 0$, we define the search direction at $x \in R^{n}$ :

$$
d_{t}=-(1-t) \nabla f(x)-t \nabla p\left(x, x^{*}, \rho\right),
$$

where $0 \leq t \leq 1$.

Theorem 8. Suppose $d_{t} \neq 0, x \in R^{n}$.

(1) If $\|\nabla f(x)\|>\left\|\nabla p\left(x, x^{*}, \rho\right)\right\|$ and $\nabla f(x)^{T} \nabla p\left(x, x^{*}, \rho\right) \geq$ $(t /(t-1))\left\|\nabla p\left(x, x^{*}, \rho\right)\right\|^{2}$, then $d_{t}$ is a descent direction of both $f(x)$ and $p\left(x, x^{*}, \rho\right)$ while $0<t<1 / 2$;

(2) if $\|\nabla f(x)\|<\left\|\nabla p\left(x, x^{*}, \rho\right)\right\|$ and $\nabla f(x)^{T} \nabla p\left(x, x^{*}, \rho\right) \geq$ $((t-1) / t)\|\nabla f(x)\|^{2}$, then $d_{t}$ is a descent direction of both $f(x)$ and $p\left(x, x^{*}, \rho\right)$ while $1 / 2<t<1$;

(3) if $\|\nabla f(x)\|=\left\|\nabla p\left(x, x^{*}, \rho\right)\right\|$, then $d_{t}$ is a descent direction of both $f(x)$ and $p\left(x, x^{*}, \rho\right)$ while $t=1 / 2$.

Proof. Let $\theta$ denote the angle between $\nabla f(x)$ and $\nabla p\left(x, x^{*}, \rho\right)$.

(1) Notice that $\|\nabla f(x)\|>\left\|\nabla p\left(x, x^{*}, \rho\right)\right\|$ and $0<t<1 / 2$. We have got

$$
\begin{aligned}
\nabla f(x)^{T} d_{t}= & -(1-t)\|\nabla f(x)\|^{2}-t \nabla f(x)^{T} \nabla p\left(x, x^{*}, \rho\right) \\
= & -(1-t)\|\nabla f(x)\|^{2} \\
& -t \cos \theta\|\nabla f(x)\|\left\|\nabla p\left(x, x^{*}, \rho\right)\right\| \\
\leq & \|\nabla f(x)\| \\
& \cdot\left[-(1-t)\|\nabla f(x)\|+t\left\|\nabla p\left(x, x^{*}, \rho\right)\right\|\right] \\
\leq & -(1-t)\|\nabla f(x)\|<0 .
\end{aligned}
$$

According to $\nabla f(x)^{T} \nabla p\left(x, x^{*}, \rho\right) \geq(t /(t-1))\left\|\nabla p\left(x, x^{*}, \rho\right)\right\|^{2}$, it is obvious that

$$
\begin{aligned}
\nabla p\left(x, x^{*}, \rho\right)^{T} d_{t}= & -(1-t) \nabla f(x)^{T} \nabla p\left(x, x^{*}, \rho\right) \\
& -t\left\|\nabla p\left(x, x^{*}, \rho\right)\right\|^{2} \leq 0
\end{aligned}
$$

holds.

(2) The proof is similar to case (1).

(3) If $t=1 / 2$, the condition $d_{t} \neq 0$ is equivalent to $\cos \theta \neq$ -1 . Consider

$$
\begin{aligned}
2 \nabla f(x)^{T} d_{t} & =-\|\nabla f(x)\|^{2}-\nabla f(x)^{T} \nabla p\left(x, x^{*}, \rho\right) \\
& =-\|\nabla f(x)\|^{2}-\cos \theta\|\nabla f(x)\|\left\|\nabla p\left(x, x^{*}, \rho\right)\right\| \\
& =-\|\nabla f(x)\|^{2}(1+\cos \theta)<0 .
\end{aligned}
$$

In the same way, we can learn $\nabla p\left(x, x^{*}, \rho\right)^{T} d_{t}<0$.

According to the above theorem, we have the following. 
Corollary 9. Let $d=-\nabla f(x) /\|\nabla f(x)\|-\nabla p\left(x, x^{*}, \rho\right) / \| \nabla p(x$, $\left.x^{*}, \rho\right) \|$; then $d$ is a descent direction of both $f(x)$ and $p\left(x, x^{*}, \rho\right)$ if and only if $d \neq 0$.

In the following, we discuss the relationship between the filter $F_{k}$ and the low level set $S_{2}\left(x^{*}\right)$.

Theorem 10. Suppose the filter set $F_{k}=\left\{x_{l} \mid 1 \leq l \leq k\right\}$. If $x_{k+1}$ can be accepted by the filter $F_{k}$ and dominates all of $x_{l}\left(\forall x_{l} \in\right.$ $\left.F_{k}\right)$, then $x_{k+1} \in S_{2}\left(x^{*}\right)$.

Proof. If there is an $x_{l} \in F_{k} \cap S_{2}\left(x^{*}\right)$, the conclusion is true since $x_{k+1}$ dominates $x_{l}$. Otherwise, the point $x^{*}$ is certainly in $F_{k}$, but $x_{k+1}$ dominates $x^{*}$. According to Definition 2 and inequation (4), $f\left(x_{k+1}\right)<f\left(x^{*}\right)$, that means $x_{k+1} \in S_{2}\left(x^{*}\right)$.

As above, we denote a global minimizer by $x^{G}$.

Theorem 11. Suppose that the current filter is $F_{k}=\left\{x_{l} \mid 1 \leq\right.$ $l \leq k\}, S_{2}\left(x^{*}\right) \neq \emptyset$. There must exist a point $x_{N} \in S_{2}\left(x^{*}\right)$ such that $x_{N}$ dominates all of $x_{l}\left(\forall x_{l} \in F_{k} \backslash G(P)\right)$ if $\rho>0$ large enough.

Proof. Two cases will be considered.

(i) $F_{k} \cap S_{2}\left(x^{*}\right)=\emptyset$, that is, $F_{k} \subseteq S_{1}\left(x^{*}\right) \bigcup\left\{x^{*}\right\}$. Then, $\forall x_{N} \in S_{2}\left(x^{*}\right)$, there is $f\left(x_{N}\right)<f\left(x_{l}\right), \forall x_{l} \in F_{k}$. Next we show

$$
p\left(x_{N}, x^{*}, \rho\right)<p\left(x_{l}, x^{*}, \rho\right), \quad \forall x_{l} \in F_{k} .
$$

Inequality (19) is precisely equivalent to

$$
-\left\|x_{N}-x^{*}\right\|^{2}+\frac{\rho}{1+\left(f\left(x_{N}\right)-f\left(x^{*}\right)\right)^{2}}<-\left\|x_{l}-x^{*}\right\|^{2}+\rho .
$$

If we set

$$
\rho>\left(D^{2}-\left\|x_{N}-x^{*}\right\|\right) \frac{1+\left(f\left(x_{N}\right)-f\left(x^{*}\right)\right)^{2}}{\left(f\left(x_{N}\right)-f\left(x^{*}\right)\right)^{2}},
$$

where $D$ is defined in expression (3), there surely will be

$$
\rho>\left(\left\|x_{l}-x^{*}\right\|^{2}-\left\|x_{N}-x^{*}\right\|\right) \frac{1+\left(f\left(x_{N}\right)-f\left(x^{*}\right)\right)^{2}}{\left(f\left(x_{N}\right)-f\left(x^{*}\right)\right)^{2}}
$$

since $\left\|x_{l}-x^{*}\right\| \leq D$. We can learn that inequality (22) is exactly equivalent to inequality (20) by simple derivation, which means inequality (19) holds.

(ii) $F_{k} \cap S_{2}\left(x^{*}\right) \neq \emptyset$. We only need to consider those points in the set $F_{k} \cap S_{2}\left(x^{*}\right)$, since the situation of the point in the set $F_{k} \cap S_{1}\left(x^{*}\right)$ is similar to case (i).

(a) If there is an $x^{G} \in F_{k}$, we will show that $x^{G}$ dominates all of $x_{l}\left(\forall x_{l} \in\left(F_{k} \backslash G(P)\right) \bigcap S_{2}\left(x^{*}\right)\right)$.

Obviously, $f\left(x^{G}\right)<f\left(x_{l}\right), \forall x_{l} \in F_{k} \backslash G(P)$.
Because $\left|F_{k}\right|$ is finite, there exists $\bar{x}_{l} \in F_{k} \bigcap S_{2}\left(x^{*}\right)$ to make $f\left(\bar{x}_{l}\right)=\min _{x_{l} \in F_{k}} f\left(x_{l}\right)$. And, according to continuity of $f(x)$, there must be $\varepsilon>0$ to make inequality

$$
\begin{aligned}
f\left(x^{G}\right)-f\left(x^{*}\right) & <f\left(x^{G}\right)+\varepsilon-f\left(x^{*}\right) \\
& <f\left(\bar{x}_{l}\right)-f\left(x^{*}\right) \leq f\left(x_{l}\right)-f\left(x^{*}\right) \\
& <0, \quad \forall x_{l} \in F_{k} \backslash G(P)
\end{aligned}
$$

hold.

Now, $\forall x_{l} \in\left(F_{k} \backslash G(P)\right) \bigcap S_{2}\left(x^{*}\right)$, from inequality (23) we have

$$
\begin{aligned}
p\left(x^{G}, x^{*}, \rho\right)-p\left(x_{l}, x^{*}, \rho\right) & \\
= & -\left\|x^{G}-x^{*}\right\|^{2}+\left\|x_{l}-x^{*}\right\|^{2} \\
& +\frac{\rho}{1+\left(f\left(x^{G}\right)-f\left(x^{*}\right)\right)^{2}}-\frac{\rho}{1+\left(f\left(x_{l}\right)-f\left(x^{*}\right)\right)^{2}} \\
< & \left(D^{2}-\left\|x^{G}-x^{*}\right\|^{2}\right)+\frac{\rho}{1+\left(f\left(x^{G}\right)-f\left(x^{*}\right)\right)^{2}} \\
& -\frac{\rho}{1+\left(f\left(x^{G}\right)+\varepsilon-f\left(x^{*}\right)\right)^{2}} .
\end{aligned}
$$

Thus, provided

$$
\begin{aligned}
\rho \geq & \left(D^{2}-\left\|x^{G}-x^{*}\right\|^{2}\right) \\
& \frac{\left[1+\left(f\left(x^{G}\right)-f\left(x^{*}\right)\right)^{2}\right]\left[1+\left(f\left(x^{G}\right)+\varepsilon-f\left(x^{*}\right)\right)^{2}\right]}{\left(f\left(x^{G}\right)-f\left(x^{*}\right)\right)^{2}-\left(f\left(x^{G}\right)+\varepsilon-f\left(x^{*}\right)\right)^{2}},
\end{aligned}
$$

holds, the inequality $p\left(x^{G}, x^{*}, \rho\right)<p\left(x_{l}, x^{*}, \rho\right)\left(\forall x_{l} \in\left(F_{k}\right)\right.$ $\left.G(P)) \bigcap S_{2}\left(x^{*}\right)\right)$ can be founded. So we have got that $x^{G}$ dominates all of $x_{l}\left(\forall x_{l} \in\left(F_{k} \backslash G(P)\right) \bigcap S_{2}\left(x^{*}\right)\right)$ if $x^{G} \in F_{k}$.

(b) $x^{G} \notin F_{k}$. Let $L_{\epsilon}=\left\{x_{N} \mid f\left(x_{N}\right)=f\left(x^{G}\right)+\varepsilon\right\}$. Obviously, $f\left(x_{N}\right)<f\left(\bar{x}_{l}\right) \leq f\left(x_{l}\right)\left(\forall x_{l} \in F_{k} \cap S_{2}\left(x^{*}\right)\right.$ based on inequality (23). Similar to the proof of (a), we can get $p\left(x_{N}, x^{*}, \rho\right)<p\left(x_{l}, x^{*}, \rho\right)$ if

$$
\begin{aligned}
\rho \geq & \left(D^{2}-\left\|x_{N}-x^{*}\right\|^{2}\right) \\
& \cdot \frac{\left[1+\left(f\left(x_{N}\right)-f\left(x^{*}\right)\right)^{2}\right]\left[1+\left(f\left(\bar{x}_{l}\right)-f\left(x^{*}\right)\right)^{2}\right]}{\left(f\left(x_{N}\right)-f\left(x^{*}\right)\right)^{2}-\left(f\left(\bar{x}_{l}\right)-f\left(x^{*}\right)\right)^{2}} .
\end{aligned}
$$

Now the filled function algorithm based on the filter technique for global optimization is listed.

Algorithm 12. Consider the following.

Step 1. Initialization: given a tolerance $\epsilon>0$, set the parameter $\delta>0, m>n$; choose an upper bound of $\rho, \bar{\rho}=10^{6}$; the scale 
Table 1: Problem 1.

\begin{tabular}{ccccc}
\hline$k$ & $x_{k}^{0}$ & $x_{k}^{*}$ & $f\left(x_{k}^{*}\right)$ & $F_{k}^{1}$ \\
\hline 1 & $(-2,1)$ & $(-1.6071,0.5686)$ & 2.1043 & $(-1.6071,-0.2314)$ \\
\hline 2 & $(-1.6071,-0.2314)$ & $(-1.7036,-0.7961)$ & -0.98945182155 & $(-0.1569,-0.5296)$ \\
\hline 3 & $(-0.1569,-0.5296)$ & $(-0.0898,-0.7127)$ & -1.0316 & $(0.0435,0.7204)$ \\
\hline
\end{tabular}

TABLe 2: Problem 2 for $n=5$.

\begin{tabular}{lllll}
\hline$k$ & $x_{k}^{0}$ & $x_{k}^{*}$ & $f\left(x_{k}^{*}\right)$ & $F_{k}^{1}$ \\
\hline 1 & $(8,8,8,8,8)$ & $(1.9899,1.9897,1.9897,1.9897,1.9898)$ & 3.1096 & $(1.0795,1.9777,1.9896,1.9896,1.9898)$ \\
\hline 2 & $(1.0795,1.9777,1.9896,1.9896,1.9898)$ & $(1.0000,1.0000,1.0000,1.0000,1.0000)$ & 0 & $(1.0000,1.0000,1.0000,1.0000,1.0000)$ \\
\hline
\end{tabular}

factor $\alpha=10$; select an initial point $x^{0} \in X$. Obtain a local minimize $x^{*}$ of $f(x)$ from $x^{0}$ by any classical method. Let the initial filter set $F_{1}=\left\{x^{*}, x^{\infty}\right\}$, where $\left\|x^{\infty}\right\|$ is large enough. Set $k=1, \rho=10$.

Step 2. $i=1$, for $i=1,2, \ldots, m$, do next.

Step 3. Choose a point $x_{k} \in U^{0}\left(x^{*}, \delta\right)$.

Step 4. If $\left|F_{k}\right|>1$, go to next step. Otherwise, go to Step 8 .

Step 5. If $\left\|\nabla f\left(x_{k}\right)\right\|>\left\|\nabla p\left(x_{k}, x^{*}, \rho\right)\right\|$, go to Step 5.1. Otherwise go to Step 6.

Step 5.1. If $\nabla f\left(x_{k}\right)^{T} \nabla p\left(x_{k}, x^{*}, \rho\right) \geq-(1 / 2)\left\|\nabla p\left(x_{k}, x^{*}, \rho\right)\right\|^{2}$, let $t=1 / 3, d^{k}=-(2 / 3) \nabla f\left(x_{k}\right)-(1 / 3) \nabla p\left(x_{k}, x^{*}, \rho\right)$; go to Step 9. Otherwise, go to Step 7.

Step 6. If $\left\|\nabla f\left(x_{k}\right)\right\|<\left\|\nabla p\left(x_{k}, x^{*}, \rho\right)\right\|$, go to Step 6.1. Otherwise go to Step 7.

Step 6.1. If $\nabla f\left(x_{k}\right)^{T} \nabla p\left(x_{k}, x^{*}, \rho\right) \geq-(1 / 2)\left\|\nabla f\left(x_{k}\right)\right\|^{2}, t=2 / 3$, $d^{k}=-(1 / 3) \nabla f\left(x_{k}\right)-(2 / 3) \nabla p\left(x_{k}, x^{*}, \rho\right)$; otherwise, $t=1$, $d_{k}=-\nabla p\left(x_{k}, x^{*}, \rho\right)$; go to Step 9 .

Step 7. Consider $d^{k}=-\nabla f(x) /\|\nabla f(x)\|-\nabla p\left(x, x^{*}, \rho\right) / \| \nabla p(x$, $\left.x^{*}, \rho\right) \|$; go to Step 9 .

Step 8. Obtain a local minimize $x^{*}$ of $f(x)$ by taking $x_{k}$ as the initial point with any classical algorithm. Go to Step 2 .

Step 9. Let $\lambda=1$.

Step 9.1. If $x_{k}+\lambda d^{k}$ can be accepted by $F_{k}$, let $x_{k+1}=x_{k}+\lambda d^{k}$; go to Step 10. Otherwise, go to next step.

Step 9.2. Let $a=0.5, \lambda=a \lambda$. If $\lambda>10^{-6}$, go to Step 9.1; otherwise, go to Step 11 .

Step 10. If $x_{k+1} \in X$, update $F_{k}$ to $F_{k+1}, k=k+1$; go to Step 4 . Otherwise, go to next step.

Step 11. $i=i+1$. If $i \leq m$; go to Step 3. Otherwise go to next step.

Step 12. If $\rho>\bar{\rho}$, the algorithm stops and $f\left(x^{*}\right)$ can be treated as the optimal value. Otherwise, $\rho=\alpha \rho$; go to Step 2 .

\section{Numerical Results}

The aim of this section is to apply filter-filled function algorithm to some classical and well known minimization problems. Based on the proposed algorithm, we use matlab $2012 \mathrm{~b}$ working on the windows 7 system with Inter3 $2328 \mathrm{M}$ CPU and $2 \mathrm{G}$ RAM. The numerical examples investigated are the following ones.

Problem 1 (6-hump camel back function [4]). Consider

$$
\begin{gathered}
f(x)=4 x_{1}^{2}-2.1 x_{1}^{4}+\frac{1}{3} x_{1}^{6}-x_{1} x_{2}-4 x_{2}^{2}+4 x_{2}^{4} \\
-3 \leq x_{1}, \quad x_{2} \leq 3 .
\end{gathered}
$$

The global minimum solutions are $x_{G}^{*}=(0.0898,0.7127)$ or $(-0.0898,-0.7127)$ and $f_{G}^{*}=-1.0316$.

Problem 2 ( $n$-dimensional Sine-square function $I$ [2]). Consider

$$
\begin{aligned}
f(x)=\frac{\pi}{n}\{1 & 10 \sin ^{2}\left(\pi x_{1}\right) \\
& +\sum_{i=1}^{n-1}\left[\left(x_{i}-1\right)^{2}\left(1+10 \sin ^{2}\left(\pi x_{i+1}\right)\right)\right] \\
& \left.+\left(x_{n}-1\right)^{2}\right\} \\
-10 & \leq x_{i} \leq 10, \quad i=1,2, \ldots, n .
\end{aligned}
$$

The function is tested for $n=5,10$. The global minimum solution is uniformly expressed as $x_{G}^{*}=(1.0000,1.0000, \ldots$, $1.0000)$ and $f_{G}^{*}=0.0000$.

The algorithm presented can also be used to solve the nonlinear system of equations

$$
F(x)=0, \quad x \in R^{n},
$$

where $F: R^{n} \rightarrow R^{m}$ is a vector function. Let $f(x)=\|F(x)\|_{2}^{2}$ in the process of calculation. 


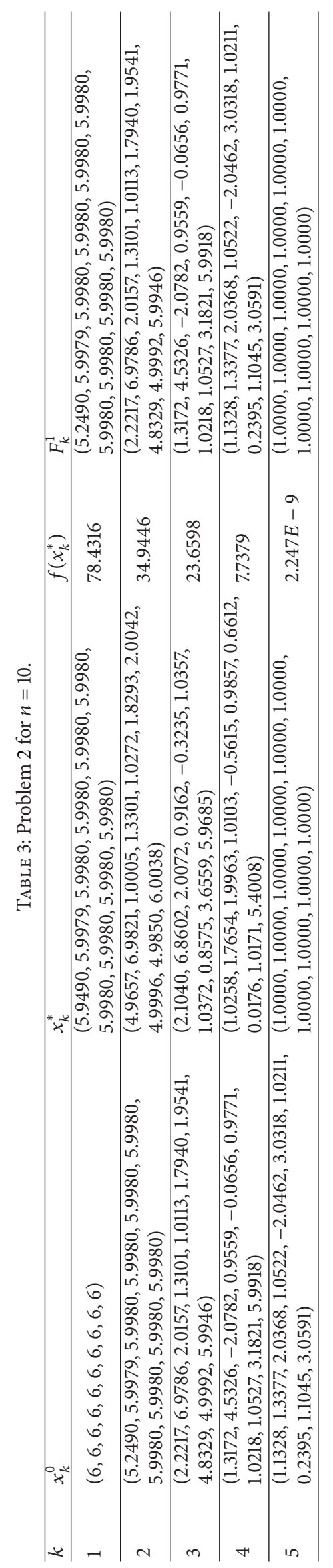


Table 4: Problem 3.

\begin{tabular}{|c|c|c|c|c|}
\hline$k$ & $x_{k}^{0}$ & $x_{k}^{*}$ & $f\left(x_{k}^{*}\right)$ & $F_{k}\left(x_{k}^{*}\right)$ \\
\hline \multirow{5}{*}{1} & 1.0000 & 0.0174 & \multirow{5}{*}{$1.197 e-004$} & 0.0072 \\
\hline & 3.0000 & 5.7093 & & -0.0053 \\
\hline & 4.0000 & 0.1582 & & 0.0040 \\
\hline & 3.0000 & 0.8555 & & 0.0039 \\
\hline & 1.0000 & 0.0365 & & $(-0.0029)$ \\
\hline \multirow{5}{*}{2} & 0.0173 & 0.0056 & \multirow{5}{*}{$5.1942 e-006$} & 0.0012 \\
\hline & 5.7094 & 18.9850 & & -0.0022 \\
\hline & 0.1583 & 0.0877 & & $7.5084 e-4$ \\
\hline & 0.8554 & 0.8593 & & 0.0013 \\
\hline & 0.0365 & 0.0369 & & $-3.2927 e-4$ \\
\hline \multirow{5}{*}{3} & 0.0055 & 0.0034 & \multirow{5}{*}{$3.2195 e-012$} & -0.0011 \\
\hline & 18.98501 & 31.3287 & & -0.0022 \\
\hline & 0.0876 & 0.0683 & & $-7.1489 e-4$ \\
\hline & 0.8593 & 0.8595 & & -0.0016 \\
\hline & 0.0369 & 0.0370 & & -0.0010 \\
\hline
\end{tabular}

TABle 5: Problem 4.

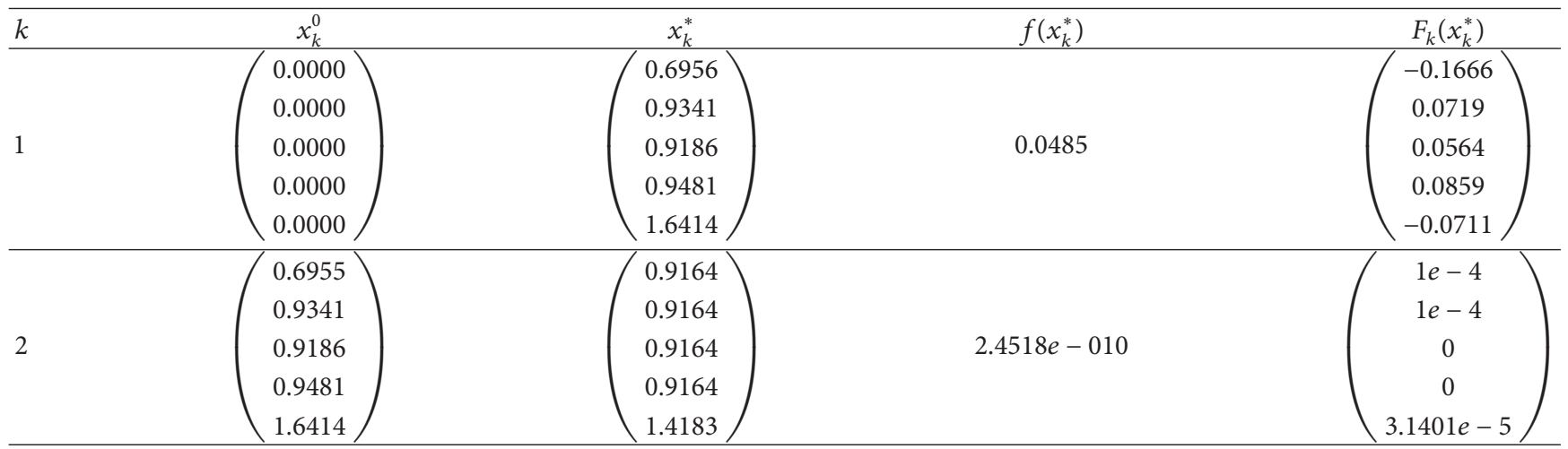

Problem 3 (see [12-14]). Consider

$$
\begin{gathered}
x_{1} x_{2}+x_{1}-3 x_{5}=0, \\
2 x_{1} x_{2}+x_{1}+3 R_{10} x_{2}^{2}+x_{2} x_{3}^{2}+R_{7} x_{2} x_{3} \\
+R_{9} x_{2} x-4+R_{8} x_{2}-R x_{5}=0, \\
2 x_{2} x_{3}^{2}+R_{7} x_{2} x_{3}+2 R_{5} x_{3}^{2}+R_{6} x_{3}-8 x_{5}=0, \\
R_{9} x_{2} x_{4}+2 x_{4}^{2}-4 R x_{5}=0, \\
x_{1} x_{2}+x_{1}+R_{10} x_{2}^{2}+x_{2} x_{3}^{2}+R_{7} x_{2} x_{3}+R_{9} x_{2} x_{4} \\
+R_{8} x_{2}+R_{5} x_{3}^{2}+R_{6} x_{3}+x_{4}^{2}-1=0, \\
0.0001 \leq x_{i} \leq 100, \quad i=1,2,3,4,5,
\end{gathered}
$$

where

$$
\begin{array}{cc}
R=10, \quad R_{5}=0.193, & R_{6}=4.10622 \times 10^{-4}, \\
R_{7}=5.45177 \times 10^{-4}, & R_{8}=4.4975 \times 10^{-7}, \\
R_{9}=3.40735 \times 10^{-5}, & R_{10}=9.615 \times 10^{-7} .
\end{array}
$$

The known solution of this problem in [14] is

$$
(0.003431,31.325636,0.068352,0.859530,0.036963)^{T} .
$$

Problem 4 (see [12-14]). Consider

$$
\begin{gathered}
2 x_{1}+x_{2}+x_{3}+x_{4}+x_{5}-6=0, \\
x_{1}+2 x_{2}+x_{3}+x_{4}+x_{5}-6=0, \\
x_{1}+x_{2}+2 x_{3}+x_{4}+x_{5}-6=0, \\
x_{1}+x_{2}+x_{3}+2 x_{4}+x_{5}-6=0, \\
x_{1} x_{2} x_{3} x_{4} x_{5}-1=0, \\
-2 \leq x_{i} \leq 2, \quad i=1,2,3,4,5 .
\end{gathered}
$$

The known solution of this problem in [14] is

$$
(1,1,1,1,1)^{T}, \quad(0.916,0.916,0.916,0.916,1.418)^{T} .
$$


Now we list the computational results. The main iterative results are summarized in Tables 1, 2, 3, 4, and 5 for each function. The symbols used are shown as follows:

$x_{k}^{0}$ : the $k$ th initial point;

$k$ : the iteration number in finding the $k$ th local minimizer;

$x_{k}^{*}$ : the $k$ th local minimizer;

$f\left(x_{k}^{*}\right)$ : the function value of the $k$ th local minimizer;

$F_{k}^{1}$ : the only entry which dominates the others in the $k$ th basin;

$F_{k}\left(x_{k}^{*}\right)$ : the vector function values at $x_{k}^{*}$ in test Problems 3 and 4 .

\section{Conflict of Interests}

The authors declare that there is no conflict of interests regarding the publication of this paper.

\section{Acknowledgment}

The authors gratefully acknowledge the supports of the National Natural Science Foundation of China (nos. 11271128 and 71372113).

\section{References}

[1] R. P. Ge and Y. F. Qin, "A class of filled functions for finding global minimizers of a function of several variables," Journal of Optimization Theory and Applications, vol. 54, no. 2, pp. 241252, 1987.

[2] R. P. Ge and Y. F. Qin, “The globally convexized filled functions for global optimization," Applied Mathematics and Computation, vol. 35, no. 2, pp. 131-158, 1990.

[3] L.-S. Zhang, C.-K. Ng, D. Li, and W.-W. Tian, "A new filled function method for global optimization," Journal of Global Optimization, vol. 28, no. 1, pp. 17-43, 2004.

[4] W. Wang and $\mathrm{Y} . \mathrm{Xu}$, "Simple transformation functions for finding better minima," Applied Mathematics Letters, vol. 21, no. 5, pp. 502-509, 2008.

[5] Y. M. Liang, L. S. Zhang, M. M. Li, and B. S. Han, "A filled function method for global optimization," Journal of Computational and Applied Mathematics, vol. 205, no. 1, pp. 1631, 2007.

[6] H. Lin, Y. Wang, L. Fan, and Y. Gao, "A new discrete filled function method for finding global minimizer of the integer programming," Applied Mathematics and Computation, vol. 219, no. 9, pp. 4371-4378, 2013.

[7] B. W. Ling, C. Z. Wu, K. L. Teo, and V. Rehbock, "Global optimal design of IIR filters via constraint transcription and filled function methods," Circuits, Systems, and Signal Processing, vol. 32, no. 3, pp. 1313-1334, 2013.

[8] R. Fletcher and S. Leyffer, "Nonlinear programming without a penalty function," Mathematical Programming, vol. 91, no. 2, pp. 239-269, 2002.

[9] R. Fletcher, S. Leyffer, and P. L. Toint, "On the global convergence of a filter-SQP algorithm," SIAM Journal on Optimization, vol. 13, no. 1, pp. 44-59, 2002.
[10] A. Wachter and L. T. Biegler, "Line search filter methods for nonlinear programming: local convergence," SIAM Journal on Optimization, vol. 16, no. 1, pp. 32-48, 2005.

[11] W. Wang, S. Hua, and J. Tang, "A generalized gradient projection filter algorithm for inequality constrained optimization," Journal of Applied Mathematics, vol. 2013, Article ID 854890, 6 pages, 2013.

[12] C. J. Wang, R. H. Luo, K. Wu, and B. S. Han, "A new filled function method for an unconstrained nonlinear equation," Journal of Computational and Applied Mathematics, vol. 235, no. 6, pp. 1689-1699, 2011.

[13] Z. Y. Wu, F. S. Bai, G. Q. Li, and Y. J. Yang, "A new auxiliary function method for systems of nonlinear equations," Journal of Industrial and Management Optimization, vol. 11, no. 2, pp. 345-364, 2015.

[14] C. A. Floudas, P. M. Pardalos, C. S. Adjiman et al., Handbook of Test Problems in Local and Global Optimization, Kluwer Academic Publishers, Dordrecht, The Netherlands, 1999. 


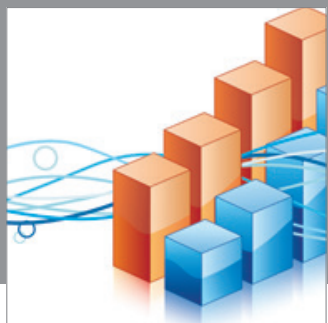

Advances in

Operations Research

mansans

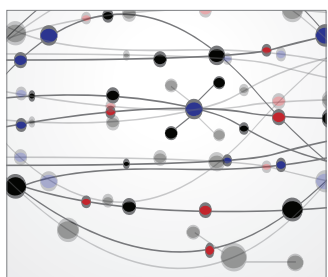

The Scientific World Journal
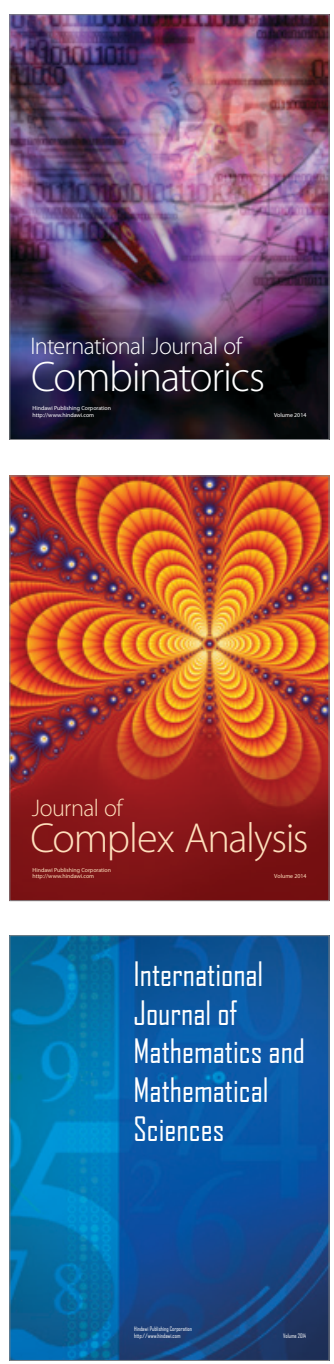
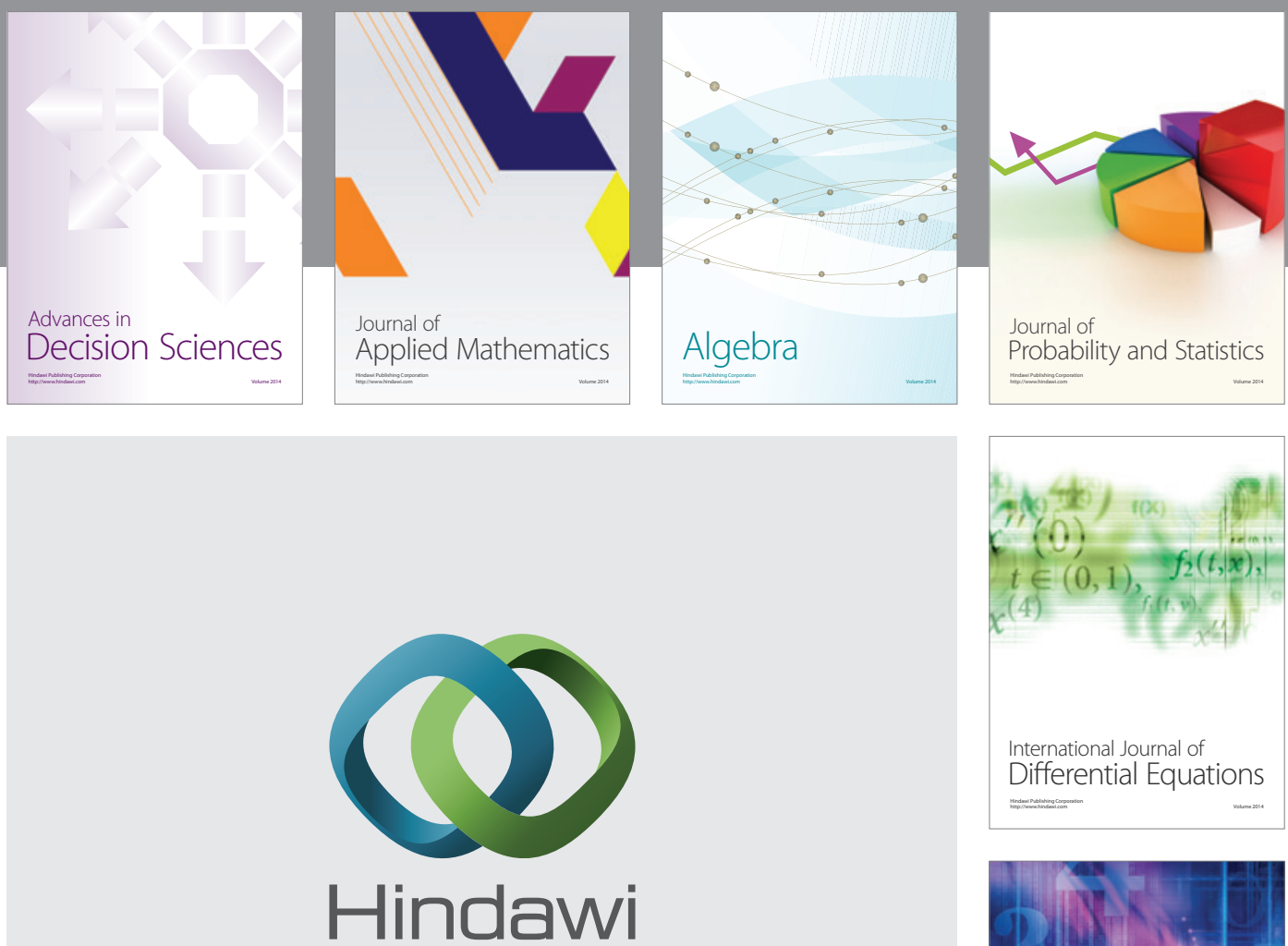

Submit your manuscripts at http://www.hindawi.com
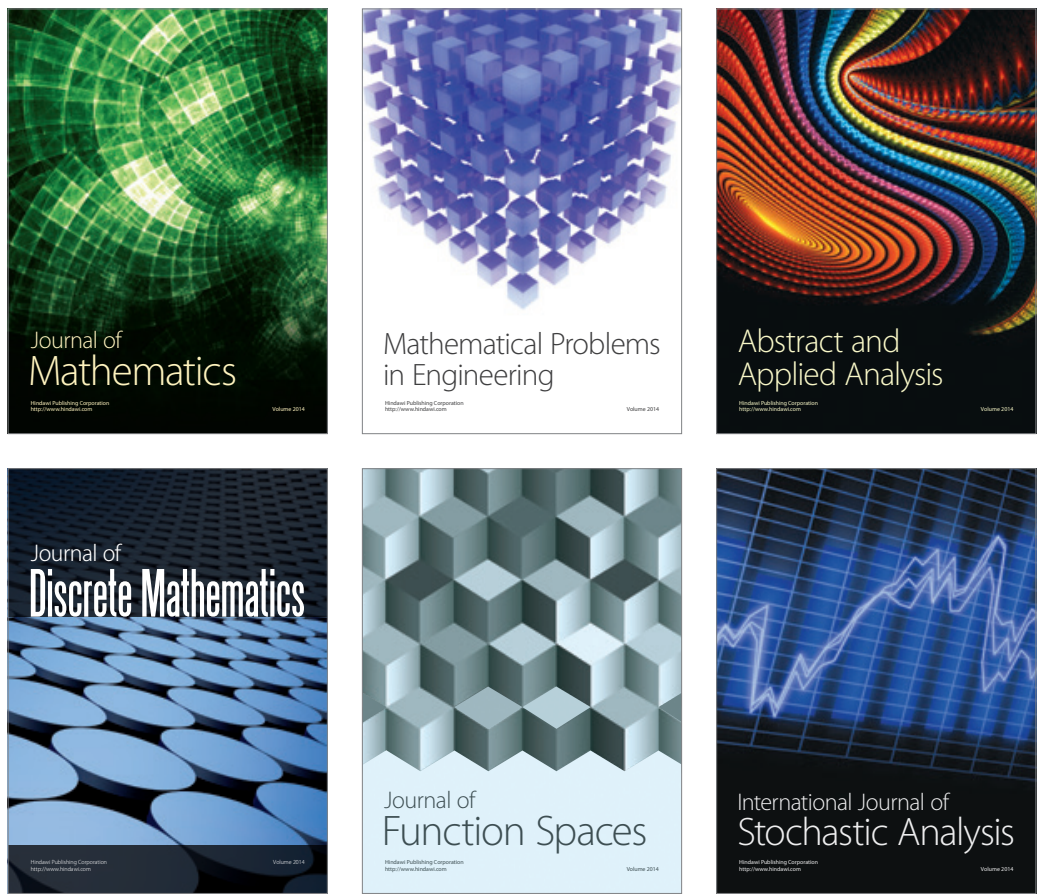

Journal of

Function Spaces

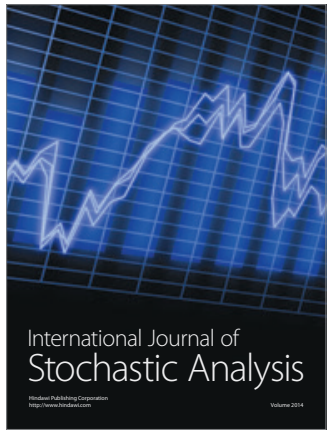

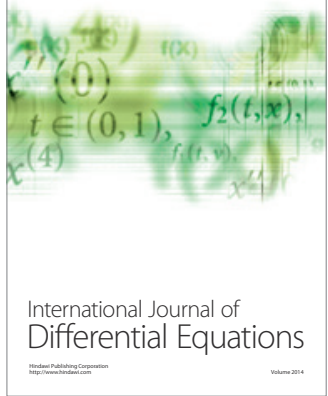
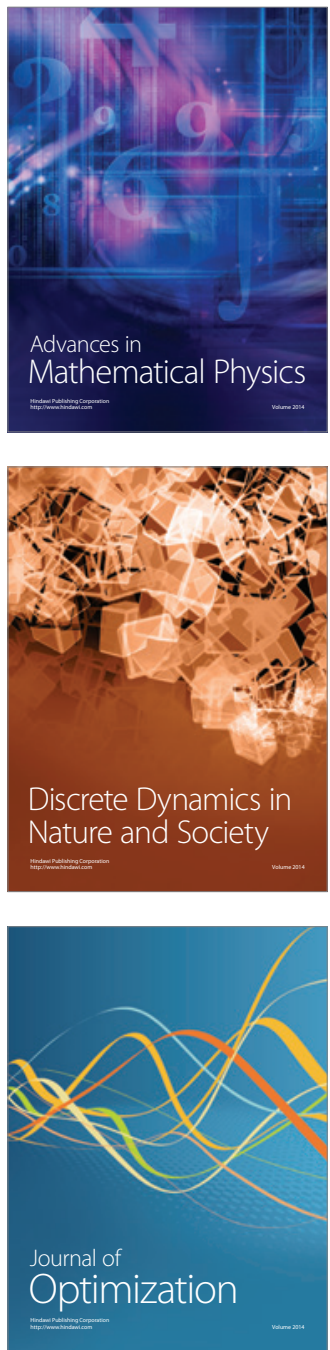